\title{
EFFETS DES HORMONES GONADOTROPES SUR L'OVAIRE DE RATTE IMMATURE
}

\author{
Marie-Madeleine DE REVIERS et P. MAULÉON \\ Station de Physiologie de la Reproduction, I. N.R. A., \\ B. P. 1 Nouzilly 37380 Monnaie (France)
}

\section{RÉSUMÉ}

Les modifications de la population folliculaire ovarienne de la ratte immature ont été mesurées quantitativement en prenant la taille du follicule comme critère de classification. Des rattes hypophysectomisées ou normales, sensibilisées par HCG, habituellement utilisées pour les dosages de FSH, ont servi de matériel animal à cette analyse biométrique.

L'action de l'hormone FSH sur la population folliculaire des ovaires des animaux utilisés dans ces deux conditions physiologiques, se situe d'abord au niveau des follicules à antrum. L'augmentation de la surface folliculaire ( $\Sigma$ taille des follicules $\times$ nombre de follicules de cette taille) est proportionnelle à la dose de FSH. Injectées séparément, GH, LH et la prolactine n'ont pas d'action sur la croissance des follicules à antrum chez la Ratte immature hypophysectomisée. Mais ils agissent sur les follicules en début de croissance. Une forte interaction entre FSH et LH existe tout au long de la croissance folliculaire.

Les actions de FSH sur la vitesse d'évolution et le taux d'involution des follicules dans les deux situations endocriniennes ne sont pas les mêmes et font l'objet de la discussion. La dépendance des différentes phases de la croissance folliculaire vis-à-vis des hormones hypophysaires est également discutée.

Une unité d'hormone folliculo-stimulante est la plus petite quantité de produit qui, injectée à une ratte immature hypophysectomisée, provoque l'apparition de follicules à antrum (" medium follicles") dans l'ovaire (Evans et al., r939).

Pour doser l'activité de cette hormone, STEELMAN et POHLEY (I953) ont proposé un test où l'augmentation du poids de l'ovaire est mesurée après l'injection de quantités croissantes de FSH et d'une quantité constante de HCG chez la Ratte immature normale.

Les conditions physiologiques sont différentes, ainsi que les critères utilisés, puisque, dans le premier cas, une seule catégorie de follicules est concernée et, dans l'autre, l'augmentation du poids ovarien résulte d'un phénomène global qui peut être aussi bien dû à un développement du follicule qu'à une lutéinisation. 
En fait, aussi précise que puisse paraître la définition de l'activité biologique de FSH, les effets des différentes préparations de cette hormone et les interactions avec LH restent ambigus. Cela est dû en particulier au fait que les critères du développement folliculaire sont seulement descriptifs (EvANs et al., I939; LosTROH et Johnson, I966) et qu'ils ne concernent que la catégorie folliculaire la plus facilement observable, celle des follicules en début de formation d'antrum.

Aussi nous a-t-il semblé nécessaire d'étudier les modifications quantitatives apportées par FSH à la totalité de la population folliculaire dans les deux types d'animaux où elle est habituellement dosée.

Cette étude de l'action de FSH sur les follicules a porté sur les principales caractéristiques des dosages biologiques : proportionnalité vis-à-vis de la dose, spécificité, interaction avec les autres hormones gonadotropes.

\section{MATÉRIEL、 ET MÉTHODES}

\section{A. - Animaux et traitements}

\section{Animaux traités selon Evans et al. (1939).}

Les conditions préconisées par ces auteurs ont été suivies. Des rattes Wistar de la souche o3 (C. R. de Nouzilly) ont été hypophysectomisées à l'âge de 27 jours par voie auriculaire selon la technique de FaLCONI et Rossi (I964). Après un temps de régression de 7 jours, les animaux ayant pris du poids pendant cette période sont considérés comme partiellement hypophysectomisés et ils sont écartés. Les autres reçoivent une injection sous-cutanée journalière pendant trois jours de $1^{\prime}$ hormone diluée dans $\mathrm{I} \mathrm{ml}$ de sérum physiologique. Le temps entre la première injection et l'abattage est de 72 heures. Les animaux sont tués par le chloroforme et les selles turciques examinées. Les surrénales sont prélevées et pesées et servent de critère supplémentaire de réussite de l'opération.

\section{Animaux traités selon Steezman et Pohley (1953).}

Les rattes sont âgées de $\mathbf{2 4}$ jours. Elles reçoivent en trois jours six injections sous-cutanées de $0,5 \mathrm{ml}$ de sérum physiologique dans lequel se trouvent diluées zo UI d'HCG et différentes doses de FSH.

Le temps entre la première injection et l'abattage est de $8_{4}$ heures.

\section{Hormones utilisées.}

Les hormones utilisées dans cette étude sont les suivantes :

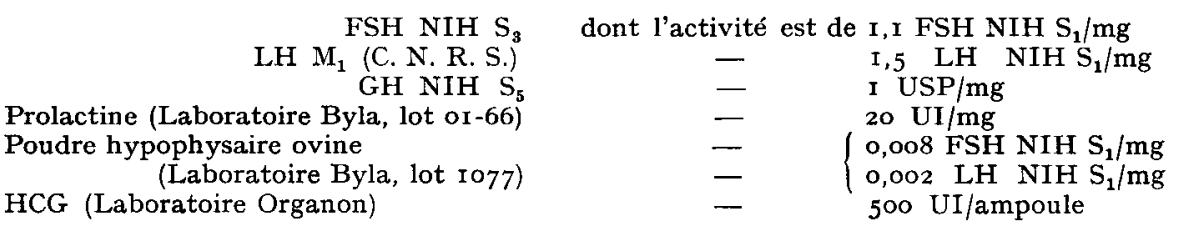

\section{B. - Techniques histologiques}

Après l'abattage des animaux les ovaires sont disséqués, pesés et fixés dans du BouinHollande. Après inclusion dans la paraffine, des coupes sont effectuées en série à ro $\mu$. Elles sont ensuite colorées par le trichrome de Masson. 


\section{C. - Méthode d'analyse de la population folliculaire}

a) Analyse de la population des follicules en croissance.

Cette technique d'analyse est voisine de celle utilisée par Mauléon et Rao (I963) et MaULÉon et BENorT (I964). Après coloration chaque coupe est projetée sur un écran à l'aide d'un microscope à projection. Le contour de la membrane basale entourant le follicule est reporté sur un papier calque quand le plan de coupe passe par le nucléole du noyau de l'ovocyte qui sert ainsi de point de repère.

Tous les follicules en croissance, d'une taille supérieure à I $259 \mu^{2}$, sont dessinés exceptés ceux qui présentent des points de pycnose dans leur granulosa et qui sont considérés comme atrétiques.

Le papier calque, de poids par $\mathrm{cm}^{2}$ homogène et connu, est alors découpé et pesé ; la surface de chaque structure folliculaire est calculée.

Les follicules sont répartis selon leur taille en classes qui sont en progression géométrique de raison I 259 à partir de I $259 \mu^{2}$. Les follicules de taille inférieure sont classés comme follicules primordiaux. La distribution des classes de taille du follicule se fait en coordonnées semi-logarithmiques.

\section{I. Établissement de la courbe de fréquence.}

La courbe de fréquence des tailles des follicules est alors établie ; un exemple est donné dans la figure I. Elle est la moyenne des tailles de follicules de deux ovaires d'animaux différents.

Dans une telle courbe, nous avons distingué arbitrairement trois zones qui nous ont semblé représenter trois paliers de la croissance du follicule :

- Zone I: Les follicules sont répartis en classes de tailles comprises entre I 259 et 5 or $2 \mu^{2}$, ils ont de I à 3 couches de cellules de granulosa.

- Zone II: Les classes de tailles sont comprises entre 5 or 2 et 50 r $20 \mu^{2}$. Les follicules ont plus de 4 couches de cellules de granulosa et l'antrum commence parfois à se former. Chez les Rattes hypophysectomisées et ne recevant pas de traitement hormonal aucun follicule ne dépasse la taille de $50120 \mu^{2}$.

- Zone III: Les follicules ont une taille supérieure à 50 I $20 \mu^{2}$. Ils possèdent un antrum plus ou moins développé.

2. Étude de la courbe de fréquence.

Pour chaque courbe de fréquence et dans chaque zone nous avons considéré :

a) le mode de distribution de la courbe;

ß) le nombre de follicules présents : le pourcentage de ces follicules est calculé par rapport aux animaux témoins ou à ceux recevant la plus faible dose d'hormone qui servent de référence I 00 p. Ioo ;

r) la surface folliculaire qui est l'intégrale des produits de toutes les tailles de follicules dans une zone donnée par le nombre de follicules dans chaque classe de taille.

b) Analyse de la population des follicules primordiaux.

Selon Mariana (I972), nous avons considéré deux groupes parmi les follicules de taille inférieure à I $259 \mu^{2}$ : aplaties,

- les follicules primordiaux où l'ovocyte est entouré d'une série discontinue de cellules

- les follicules ayant débuté leur croissance : l'ovocyte est entouré d'une couche continue de cellules cuboïdes.

Les follicules dont le noyau de l'ovocyte est présent dans une coupe sont comptés dans la totalité de cette coupe. Un échantillonnage d'une coupe sur ro est réalisé et le nombre total de follicules est calculé en fonction du nombre total de coupes et corrigé selon ABERCROMBIE (1946). 


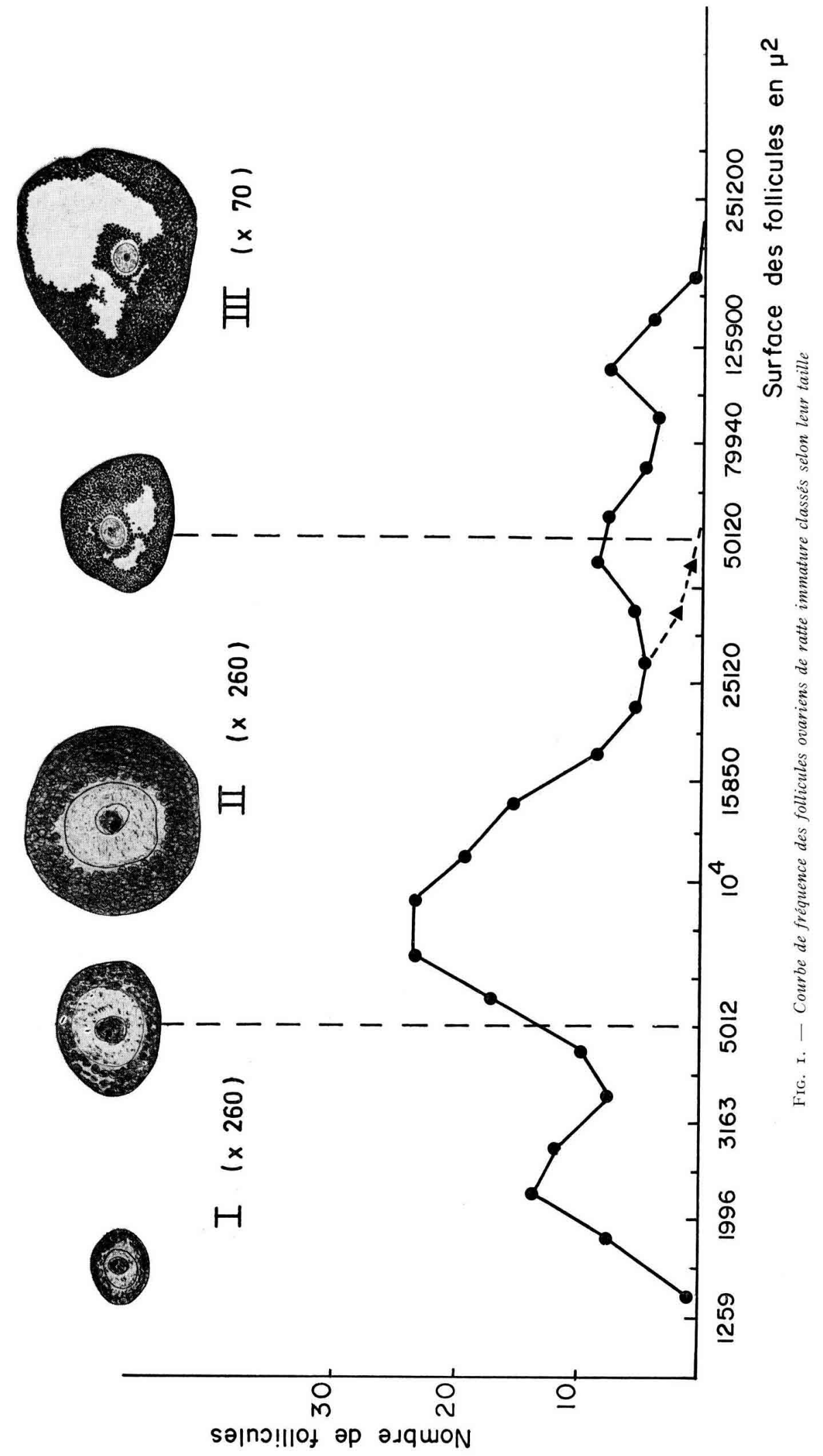




\section{RÉSULTATS}

\section{A. - Effets de FSH sur le poids de l'ovaire}

et sur la population folliculaire de la Ratte normale et hypophysectomisée.

Proportionnalité de la réponse

a) Effet sur le poids des ovaires.

Chez la Ratte hypophysectomisée et traitée dans les conditions de Evans et al. (1939), des doses croissantes de FSH provoquent une augmentation du poids de l'ovaire proportionnelle au logarithme de la dose injectée. Il en est de même chez l'animal normal traité selon Strezlman et Pohley (1953) (tabl. r), mais il est net sur la figure 7 que la pente de la droite de réponse est beaucoup plus forte dans ce cas.

\section{TABLEAU I}

Effet de doses croissantes de FSH sur le poids ovarien de la ratte normale et de la ratte hypophysectomisée

\begin{tabular}{|c|c|c|c|c|}
\hline \multirow{2}{*}{ Type d'animal } & \multicolumn{4}{|c|}{ Poids ovarien $\left(\mathrm{mg} \pm s_{m}\right)$ pour les doses de FSH $(\mu \mathrm{g})$ : } \\
\hline & 0 & 80 & 160 & 320 \\
\hline $\begin{array}{c}\text { Hypophysectomisé } \\
\text { (Evans) }\end{array}$ & $7,2 \pm 0,7$ & 12,0 & $21,7 \pm 0,5$ & 32,1 \\
\hline $\begin{array}{c}\text { Normal } \\
\text { (Steelman et Pohley) }\end{array}$ & $40,5 \pm 3,5$ & $68,0 \pm 8,4$ & $150,0 \pm 3 z^{\prime}, \mathbf{t}$ & $178,7 \pm 23,2$ \\
\hline
\end{tabular}

b) Effet sur la population folliculaire.

I. Population folliculaire des deux types d'animaux témoins.

Dix jours après l'hypophysectomie, la population folliculaire subit des changements étudiés par BENOIT ( 1967 ) : le nombre de follicules normaux diminue de $30 \mathrm{p}$. Ioo dans la zone II et tous les follicules de taille supérieure à 50 I2O $\mu^{2}$ sont devenus atrétiques.

La comparaison des courbes de fréquence des tailles de follicules de ratte traitée avec 20 UI d'HCG ou de ratte normale du même âge fait apparaître chez les animaux traités, une diminution du nombre de follicules entre les classes I 259 et ro o0o $\mu^{2}$; au-delà de cette taille la courbe présente peu de changements (fig. 2).

2. Populations folliculaires des animaux traités avec des doses croissantes de FSH.

$\alpha$. Les courbes de fréquence (fig. 3) de la population folliculaire des ovaires de rattes hypophysectomisées et traitées avec des doses croissantes de FSH subissent des modifications frappantes :

- une augmentation par rapport aux animaux témoins du nombre de follicules 


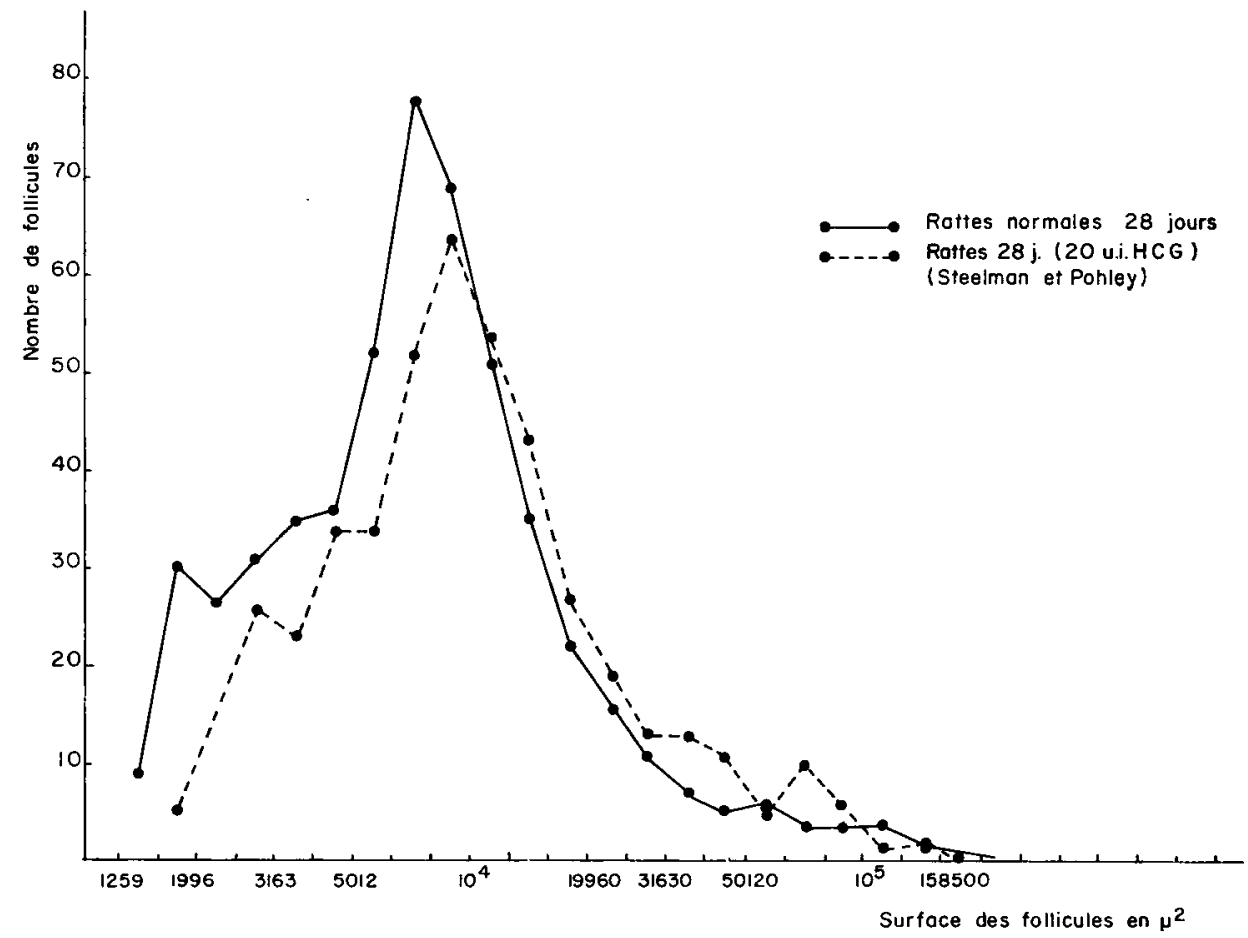

FIG. 2. - Comparaison des populations folliculaires de rattes normales et traitées par $H C G$

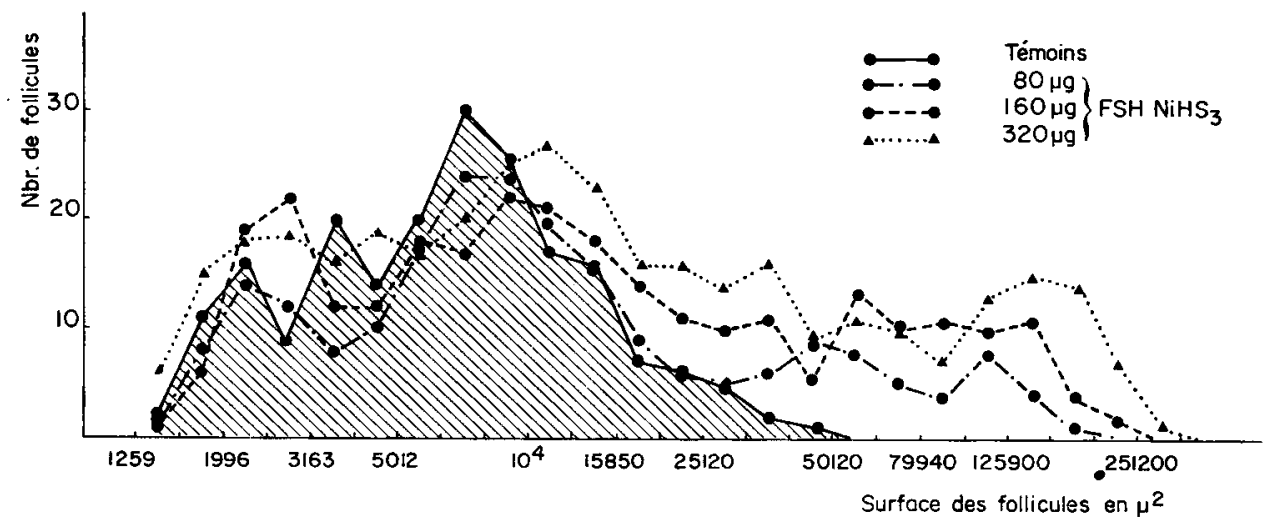

FIG. 3. - Effet de doses croissantes de FSH

sur la population folliculaire de la ratte immature hypophysectomisée

présents, peu marquée dans la zone I, mais importante dans la zone II (tabl. 2) pour les plus fortes doses de FSH,

- l'apparition d'une population nouvelle de follicules de taille supérieure à $50000 \mu^{2}$,

- un déplacement, de l'ordre de deux classes de tailles, du mode de la courbe vers les plus grandes tailles de follicules dans la zone II, 
- une augmentation de la surface folliculaire, particulièrement dans les zones II et III. Dans cette troisième zone, l'augmentation de la surface folliculaire stimulée est proportionnelle au logarithme de la dose de FSH injectée (tabl. 3).

TABLEAU 2

Variations relatives $d u$ nombre de follicules appartenant à chacune des trois zones de la courbe de fréquence des ovaires de rattes hypophysectomisées traitées par $F S H$

\begin{tabular}{|c|c|c|c|}
\hline \multirow{2}{*}{$\begin{array}{c}\text { Doses de FSH } \\
(\mu g)\end{array}$} & \multicolumn{2}{|c|}{ Nombre de follicules : } & $\frac{\text { Ovaires traités }}{\text { Ovaires témoins }}$ \\
\hline & Zone 1 & Zone II & Zone III \\
\hline 0 & 100 & 100 & 0 \\
\hline 80 & 73 & 103 & 100 \\
\hline 160 & 100 & 114 & 263 \\
\hline 320 & 126 & $1 \div 1$ & 331 \\
\hline
\end{tabular}

TABLEAU 3

Effet de doses croissantes de FSH sur les trois zones de la courbe de fréquence dans l'ovaire de ratte hypophysectomisée

\begin{tabular}{|c|c|c|c|}
\hline \multirow{2}{*}{$\begin{array}{c}\text { Doses de FSH } \\
(\mu g)\end{array}$} & \multicolumn{3}{|c|}{$\Sigma$ (Nombre de follicules $\times$ tailles) $\left(\mu^{2}\right)$} \\
\hline & Zone I & Zone II & Zone III \\
\hline 0 & 190000 & 1290000 & 0 \\
\hline 80 & 126000 & 1720000 & 1520000 \\
\hline 160 & 187000 & 2000000 & 5480000 \\
\hline 320 & 233000 & 2600000 & 8600000 \\
\hline
\end{tabular}

\section{TABLEAU 4}

Variations relatives $d u$ nombre de follicules appartenant à chacune des trois zones de la courbe de fréquence des ovaires de rattes normales traitées par FSH

\begin{tabular}{|c|c|c|c|}
\hline \multirow{2}{*}{$\begin{array}{c}\text { Doses de FSH } \\
(\mu g)\end{array}$} & \multicolumn{3}{|c|}{ Nombre de follicules : $\frac{\text { Ovaires traités }}{\text { Ovaires témoins }}$} \\
\hline & Zone I & Zone II & Zone III \\
\hline 0 & 100 & 100 & 100 \\
\hline 80 & 125 & 91 & 250 \\
\hline 160 & 100 & 87 & 505 \\
\hline 320 & 100 & 86 & 810 \\
\hline
\end{tabular}




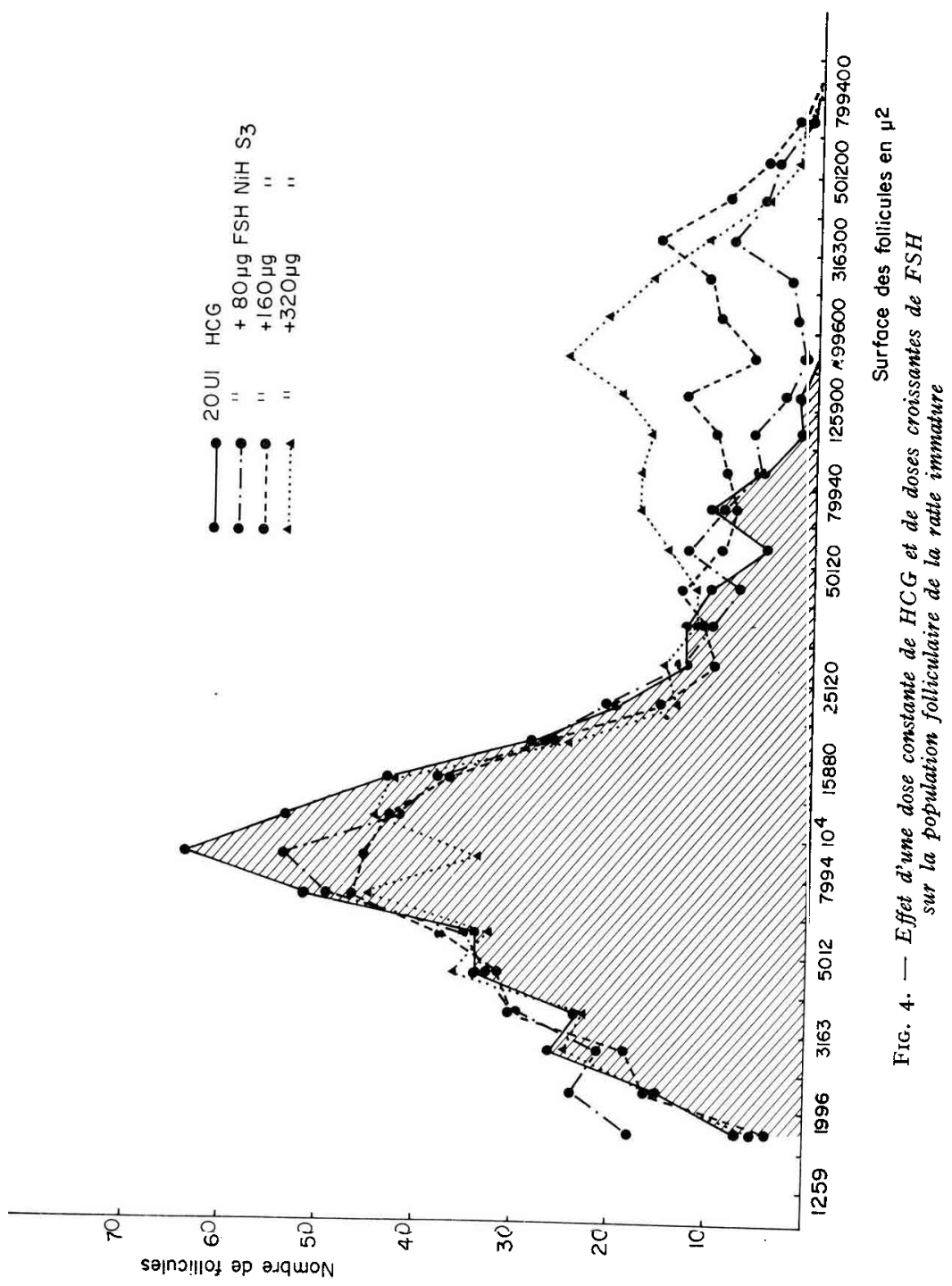


TABLEAU 5

Effet de doses croissantes de FSH (+ 20 UI HCG) sur les trois zones de la courbe de fréquence dans l'ovaire de la ratte normale

\begin{tabular}{|c|c|c|c|}
\hline \multirow{2}{*}{$\begin{array}{c}\text { Doses de FSH } \\
(\mu g)\end{array}$} & \multicolumn{3}{|c|}{$\Sigma$ (Nombre de follicules $\times$ tailles) $\left(\mu^{2}\right)$} \\
\hline & Zone I & Zone II & Zone III \\
\hline 0 & 470000 & 4000000 & 1400000 \\
\hline 80 & 540000 & 3900000 & 10200000 \\
\hline 160 & 480000 & 3800000 & 21300000 \\
\hline 320 & 475000 & 4100000 & 26500000 \\
\hline
\end{tabular}

$\beta$. Les follicules ovariens des rattes normales traitées selon STEELMAN et POHLEY avec des doses croissantes de FSH présentent une courbe de fréquence de leur taille, modifiée par rapport à celle des témoins (fig. 4).

Fille s'apparente à la courbe de distribution des tailles de follicules de l'ovaire de ratte hypophysectomisée après stimulation par FSH par :

- un accroissement important du nombre de follicules dans la zone III (tabl. 4),

- une augmentation de la surface folliculaire dans cette zone qui est proportionnelle au logarithme de la dose de FSH injectée (tabl. 5).

Mais elle en diffère par :

- une diminution du nombre de follicules dans la zone II (tabl. 4), bien que le nombre total de follicules soit plus élevé que chez l'animal hypophysectomisé,

- le développement d'une taille maximale beaucoup plus élevée des follicules de la zone III.

Enfin, le mode de la courbe ne subit pas de déplacement.

L'hormone folliculo-stimulante a donc bien une action sur les follicules à antrum chez les deux types d'animaux mais ces follicules réapparaissent chez la Ratte hypophysectomisée tandis qu'il s'agit d'une augmentation de leur nombre chez l'animal entier sensibilisé par HCG. L'approvisionnement en follicules de ce type n'entraîne pas les mêmes modifications sur les autres catégories.

\section{B. - Spécificité de la réponse}

Des rattes hypophysectomisées ont reçu les doses de $80 \mu \mathrm{g}$ de LH ou $500 \mu \mathrm{g}$ de GH ou 20 UI de prolactine sans que le poids des ovaires ne soit modifié par rapport aux témoins pour aucune de ces hormones.

Les courbes de fréquence des follicules des animaux traités avec ces hormones diffèrent peu par rapport à celles des animaux témoins (fig. 5).

Cependant :

- le nombre de follicules de la zone I augmente chez les animaux traités par l'hormone de croissance et la prolactine de l'ordre de $30 \mathrm{p}$. Ioo, 
- le mode de la courbe de fréquence se déplace dans la zone II après traitement par la prolactine, mais aucun follicule de taille supérieure à 50 ooo $\mu^{2}$ n'apparaît.
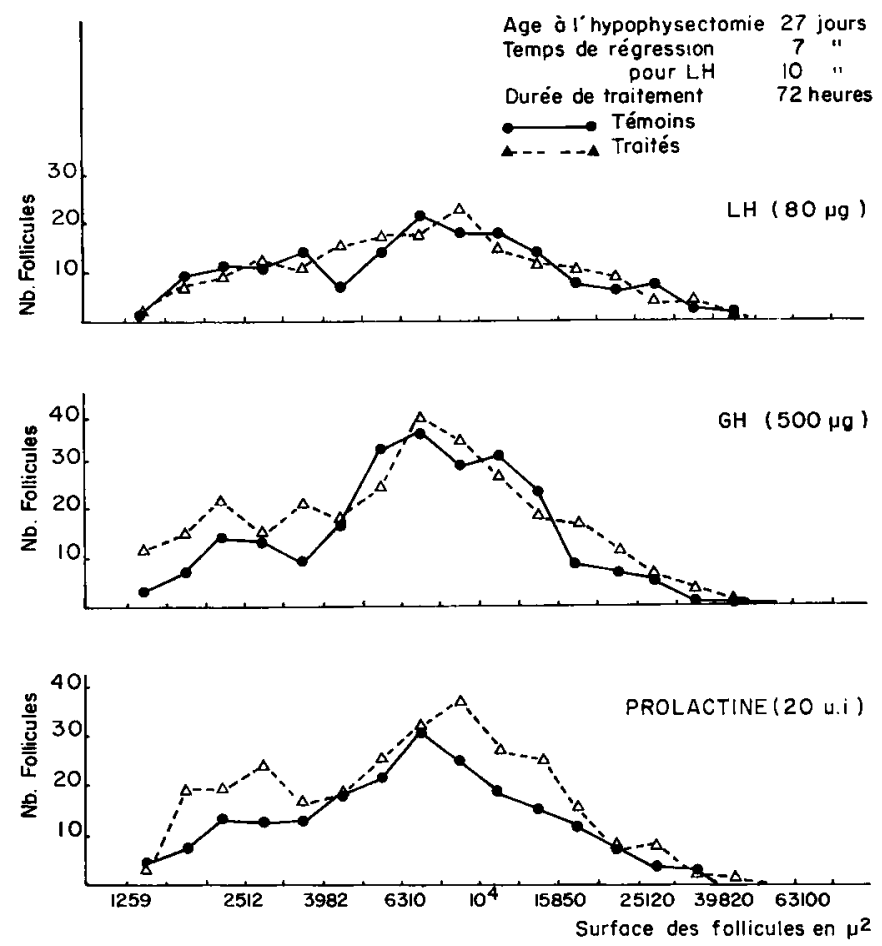

FIG. 5. - Effet de différentes hormones hypophysaires ovines sur la population folliculaire de la ratte immature hypophysectomisée

Injectées séparément chez la Ratte hypophysectomisée, les hormones hypophysaires $\mathrm{LH}, \mathrm{GH}$ et prolactine n'ont donc pas d'action sur l'apparition de follicules de taille supérieure à 50 ooo $\mu^{2}$ et sur la formation de l'antrum, mais une action est décelable au niveau des plus petits follicules.

\section{TABLEAU 6}

Accroissement dà à $L H, H C G$ ou à une poudre hypophysaire de la réponse ovarienne à FSH

\begin{tabular}{|c|c|c|c|c|}
\hline \multirow{2}{*}{ Traitement } & \multicolumn{4}{|c|}{$\begin{array}{l}\left.\text { Poids des ovaires (mg } \pm s_{m}\right) \\
\text { pour les doses de FSH }(\mu g) \text { : }\end{array}$} \\
\hline & $\mathbf{0}$ & 80 & 160 & 320 \\
\hline $0 \ldots \ldots$ & $7,2 \pm 0,7$ & 12,0 & $21,7 \pm 0,5$ & 32,1 \\
\hline $\mathrm{L} / \mathrm{H} 80 \mu \mathrm{g} \ldots$ & 9,2 & $21,8 \pm 3,4$ & $34,6 \pm 3,4$ & $31,7 \pm 2,8$ \\
\hline HCG 20 UI $\ldots \ldots$ & $15,2 \pm 0,6$ & $43,2 \pm 4,5$ & $51,5 \pm 4,8$ & $60,2 \pm 4,8$ \\
\hline Poudre hypophysaire. & $10,1 \pm 0,5$ & $24,0 \pm 1,6$ & $45,9 \pm 4,8$ & $50,6 \pm 5,4$ \\
\hline
\end{tabular}


TABLEAU 7. - Variations relatives du nombre de follicules appartenant à chacune des trois zones de la courbe de fréquence des ovaires de rattes hypophysectomisées traitées par FSH plus $L H$ ou une poudre hypophysaire

\begin{tabular}{|c|c|c|c|}
\hline \multirow{2}{*}{ Traitement } & \multicolumn{3}{|c|}{ Nombre de follicules : $\frac{\text { Ovaires traités }}{\text { Ovaires témoins }}$} \\
\hline & Zone I & Zone II & Zone III \\
\hline $\begin{array}{l}80 \mu \mathrm{g} \text { FSH } \ldots \ldots \ldots \ldots \ldots \ldots \ldots \\
80 \mu \mathrm{g} \text { FSH }+80 \mu \mathrm{L} \text { LH } \ldots \ldots \ldots \ldots \\
\text { é. } 80 \mu \mathrm{F} \text { FSH poudre hypophys } \\
\text { aire } \ldots \ldots \ldots \ldots \ldots \ldots \ldots \ldots\end{array}$ & $\begin{array}{l}100 \\
156 \\
169\end{array}$ & $\begin{array}{l}100 \\
176 \\
119\end{array}$ & $\begin{array}{l}100 \\
250 \\
178\end{array}$ \\
\hline $\begin{array}{l}80 \mu \mathrm{g} \text { FSH } \ldots \ldots \ldots \ldots \ldots \ldots \\
160 \mu \mathrm{g} \text { FSH }+80 \mu \mathrm{g} \text { LH } \ldots \ldots \ldots \ldots \\
\text { éq. } 160 \mu \mathrm{g} \text { FSH poudre hypophy- } \\
\text { saire } \ldots \ldots \ldots \ldots \ldots \ldots \ldots \ldots\end{array}$ & $\begin{array}{r}100 \\
95\end{array}$ & $\begin{array}{l}100 \\
134 \\
128\end{array}$ & $\begin{array}{l}100 \\
137 \\
143\end{array}$ \\
\hline
\end{tabular}

TABLEAU 8. - Modification par FSH du taux de cellules incorporant la thymidine tritiée dans les follicules de ratte immatuve hypophysectomisée

\begin{tabular}{|c|c|c|c|c|}
\hline \multirow{2}{*}{$\begin{array}{l}\text { Classes de follicules } \\
\text { Nombre de cellules } \\
\text { de granulosa }\end{array}$} & \multicolumn{4}{|c|}{$\frac{\text { Nombre de cellules marquées }}{\text { Nombre total de cellules }} \times 100$} \\
\hline & Tém & is $\mathrm{NaCl}$ & 160 & g FSH \\
\hline $\begin{array}{c}10-20 \\
20-40 \\
40-80 \\
80-160 \\
160-320 \\
320-640 \\
640-1280 \\
>1280\end{array}$ & $\begin{array}{r}4,9 \\
5,4 \\
20,5 \\
21,3 \\
21,9 \\
30,7 \\
30,3\end{array}$ & $\begin{array}{l}n^{*}=19 \\
n=23 \\
n=12 \\
n=16 \\
n=16 \\
n=13 \\
n=4\end{array}$ & $\begin{array}{r}9,0 \\
17,6 \\
32,0 \\
39,5 \\
33,8 \\
33,0 \\
39,9 \\
39,0\end{array}$ & $\begin{array}{l}n=15 \\
n=18 \\
n=8 \\
n=9 \\
n=19 \\
n=17 \\
n=10 \\
n=5\end{array}$ \\
\hline
\end{tabular}

$n^{*}$ : nombre de follicules examinés dans leur plus grande surface de coupe.

TABLEAU 9. - Effet de différentes hormones hypophysaires et d'une poudre hypophysaive sur le pourcentage de follicules en début de croissance par rapport au nombre total de follicules primordiaux

\begin{tabular}{|c|c|c|}
\hline \multirow{2}{*}{ Hormones injectées } & \multicolumn{2}{|c|}{$\frac{\text { Nombre de follicules en croissance }}{\text { Nombre total de follicules primordiaux }} \times 100$} \\
\hline & Ovaires témoins & Ovaires traités \\
\hline FSH $160 \mu \mathrm{g} \ldots \ldots \ldots \ldots \ldots \ldots \ldots \ldots$ & 25,0 & 29,8 \\
\hline FSH $320 \mu \mathrm{g} \ldots \ldots \ldots \ldots \ldots \ldots \ldots$ & 25,0 & 39,1 \\
\hline GH $500 \mu \mathrm{g} \ldots \ldots \ldots \ldots \ldots \ldots \ldots$ & 24,0 & 26,9 \\
\hline LH $80 \mu \mathrm{g} \ldots \ldots \ldots \ldots \ldots \ldots \ldots$ & 33,5 & 38,4 \\
\hline Prolactine 20 UI $\ldots \ldots \ldots \ldots \ldots \ldots$ & 35,1 & 37,9 \\
\hline Poudre acétonique éq. $160 \mu \mathrm{F}$ FSH .... & 35,1 & 48,9 \\
\hline
\end{tabular}




\section{C. - Augmentation de la réponse à FSH chez la Ratte hypophysectomisée après traitement par $L H, H C G$ ou une poudre hypophysaire}

Une dose de $80 \mu \mathrm{g}$ de LH ou 20 UI d'HCG ou des doses croissantes d'une poudre hypophysaire brute provoquent une augmentation du poids ovarien plus élevée que celle obtenue avec FSH seule (tabl. 6).

Les courbes de distribution des tailles de follicules des ovaires de rattes traitées avec FSH plus LH sont pratiquement homothétiques de celles obtenues avec FSH seule (fig. 6).

Le nombre des follicules présents dans toutes les classes de taille des trois zones est considérablement augmenté (tabl. 7). Cette augmentation est particuliè-
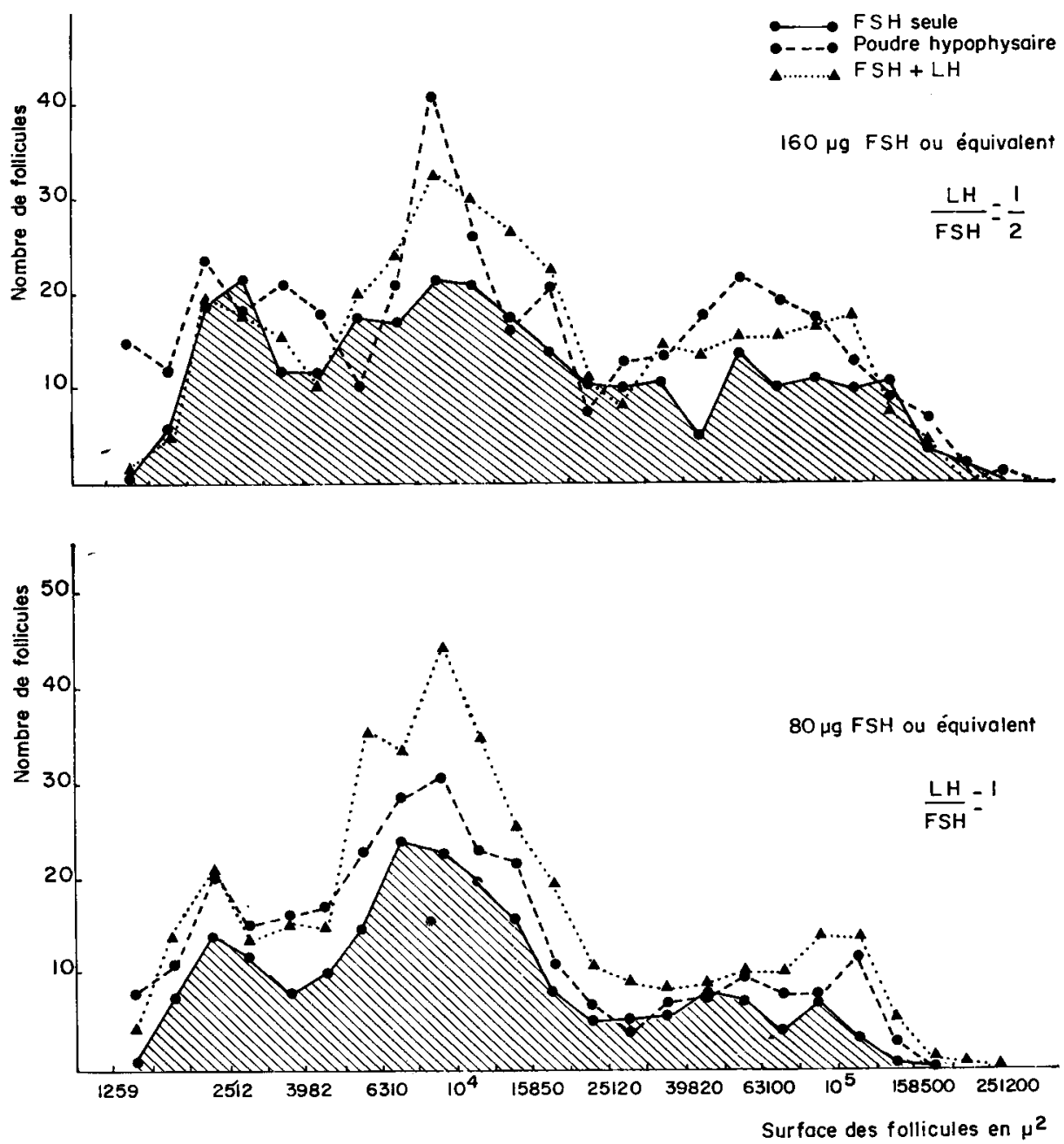

Fig. 6. - Effet de FSH seule, FSH + LH ou d'une poudre hypophysaire ovine sur la population folliculaire de la ratte immature hypophysectomisée 
rement remarquable lorsque le rapport $\frac{\mathrm{LH}}{\overline{\mathrm{FSH}}}$ est égal à I ou pour une poudre hypophysaire dont le rapport $\frac{\mathrm{LH}}{\mathrm{FSH}}$ est égal à $\frac{\mathrm{I}}{4}$.

La nature de la réponse folliculaire à FSH après interaction d'autres hormones n'est pas modifiée mais il y a augmentation de cette réponse.

\section{DISCUSSION}

Les dosages de Stemlman et Pohley (I953) et d'Evans et al. (I939) sont une mesure de l'effet biologique de 1'hormone FSH sur les follicules à antrum ou appartenant à la zone III de notre terminologie. En effet, nous avons pu vérifier que la mesure du poids ovarien correspond au développement folliculaire de la zone III (fig. 7). FSH est donc bien l'hormone qui fait apparaître l'antrum dans le follicule en croissance comme l'avaient déjà confirmé LosTroH et JoHnson (I966). Cette

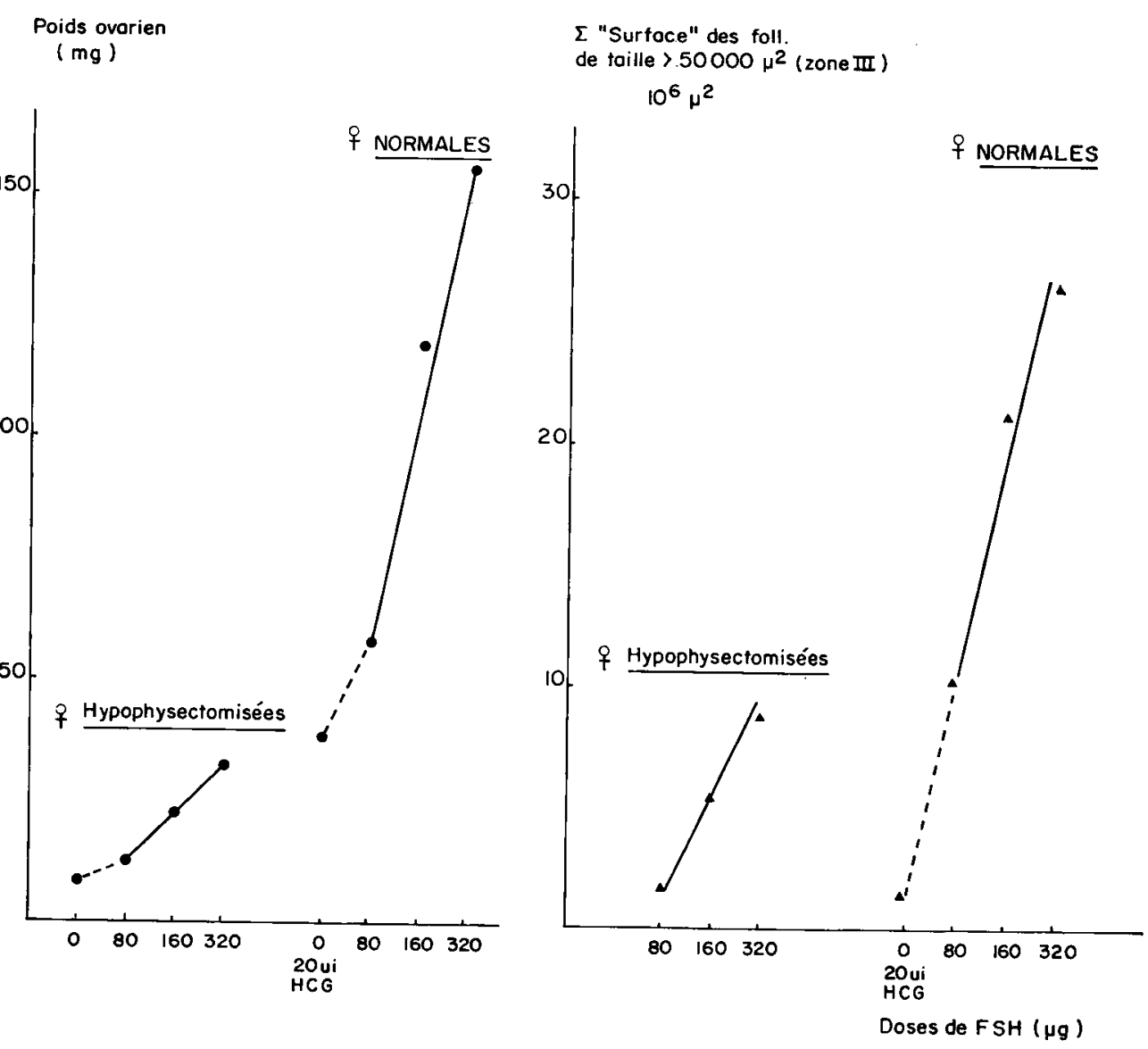

FIG. 7. - Comparaison des courbes de réponse des poids ovariens

et de la "surface " totale des plus gros follicules de l'ovaire

après traitement par des doses croissantes de FSH chez des animaux normaux et hypophysectomisês 
action est spécifique de FSH puisqu'aucun follicule de cette catégorie III n'apparaît chez l'animal immature hypophysectomisé après injection de $\mathrm{L} H, \mathrm{GH}$ et prolactine. Mais cette action concerne une phase particulière de la croissance folliculaire. Or, la croissance d'un follicule comporte plusieurs phases (PEDERSEN, I970) ou paliers correspondant à chacune des "zones" définies dans la courbe de fréquence des tailles de follicules.

En étudiant les modifications quantitatives de ces zones, nous pouvons mettre en évidence les effets des hormones sur les différentes phases de la croissance du follicule.

L'accroissement rapide de taille du follicule qui précède l'ovulation chez l'animal adulte serait représenté avec notre méthode d'analyse par des follicules de taille comprise entre 300000 et $375000 \mu^{2}$ (une zone IV selon notre terminologie). De tels follicules n'ont jamais été observés chez l'animal hypophysectomisé après injection de FSH. Par contre, une telle taille est largement dépassée chez la Ratte immature sensibilisée par HCG recevant FSH. Mais dans ce cas, le follicule est anormal car il présente un antrum qui peut atteindre $80 \mathrm{p}$. Ioo du volume total du follicule. Ce paramètre pour un follicule préovulatoire est de $45 \mathrm{p}$. Ioo. Aussi est-il difficile, d'après les résultats obtenus dans nos conditions expérimentales, de dire si FSH a une action sur cette phase de la croissance folliculaire chez la Ratte adulte cyclique.

Au contraire, les hormones FSH et surtout FSH + LH injectées à des rattes d'Evans augmentent le nombre des follicules des zones I et II, qui sont de plus petite taille que celles des follicules à antrum. Mais cette action de FSH n'est pas spécifique car l'hormone de croissance $\mathrm{GH}$ et la prolactine augmentent aussi le nombre de ces follicules, principalement ceux de la zone I. Dans les follicules de ces deux zones I et II, nous avons effectué le comptage des cellules marquées dans les follicules d'ovaires de ratte hypophysectomisées ayant reçu de la thymidine tritiée $(2 \mu \mathrm{Ci} / \mathrm{g}) 72$ heures avant l'abattage. Le nombre de cellules qui incorporent la thymidine augmente après traitement par FSH dans toutes les classes de follicules, particulièrement les plus petites. Ceci indique un taux plus élevé des multiplications cellulaires. Les résultats préliminaires de ces comptages sont donnés dans le tableau 8. Ils sont comparables à ceux de RYLE (I972) qui a trouvé une augmentation de l'incorporation de thymidine tritiée, particulièrement dans les follicules de 3 à 4 couches de granulosa mais aussi dans les follicules à I couche à la suite d'addition de FSH au milieu de culture des ovaires.

Notre méthode d'analyse ne permet pas de suivre les effets des hormones hypophysaires sur les follicules primordiaux de taille inférieure à I $259 \mu^{2}$. Ces follicules ont une seule couche de cellules rondes et sont marqués en phase $S$ du cycle cellulaire après injection de thymidine tritiée à la ratte (MARIANA, I972). Ils doivent donc être considérés comme ayant débuté leur croissance. Nous les avons dénombrés chez les rattes soumises aux divers traitements. Le rapport du nombre de follicules en début de croissance au nombre total de follicules primordiaux a été pris comme critère d'une initiation de croissance folliculaire. Il apparaît dans le tableau 9 qu'une forte dose de FSH ou une poudre hypophysaire totale peut stimuler cette initiation. Enfin, il a été montré par BAKER et NEAL, (I972) que les hormones hypophysaires jouent un rôle dans l'édification même du follicule, c'est-à-dire dans l'organisation des cellules de la granulosa autour de l'ovocyte.

Nous pouvons donc dire que contrairement à ce qu'affirmait HISAW (I947), la 
folliculogenèse dans sa totalité chez les rattes de 23 jours est sous la dépendance d'un environnement hormonal hypophysaire. FSH semble agir d'un bout à l'autre de la croissance du follicule lorsque l'on prend la taille du follicule comme critère de cette croissance. FSH a une action spécifique sur l'apparition de l'antrum. Cette action suffit à expliquer que FSH agit sur la taille du follicule tout au long de son développement, si on la considère comme une action sur la fonction de sécrétion des cellules folliculaires. En effet, cette sécrétion en accroissant les espaces cellulaires et en provoquant 1'accumulation de liquide folliculaire augmente par là même la taille des follicules. En fait, FSH agit également sur le taux de multiplication des cellules folliculeuses qui est le facteur principal d'accroissement de taille des follicules des zones I et II.

Les interprétations que nous avons données précédemment résultent des modifications absolues ou relatives des nombres de follicules d'une zone donnée, observées entre individus, une sorte de comparaison verticale des changements observables. Mais on peut également étudier les changements de forme entre les deux types de rats (STEElman-Pohley, I953 et Evans et al, I939) des courbes de fréquence des tailles de follicules considérées dans leur ensemble, une sorte de comparaison horizontale. L'interprétation de telles modifications se traduira en terme de passage des follicules d'une zone vers une autre, c'est-à-dire d'un compartiment vers un autre compartiment. Elle nous renseignera sur l'effet des hormones sur la dynamique folliculaire dans le laps de temps de 4 jours, situé entre le début des injections et l'abattage. Dans tous les cas, après injection de FSH, quel que soit le type d'animal utilisé, il y a formation d'un pic dans la zone III de la courbe; on peut se demander d'où viennent ces follicules en regardant comment se modifie le pic de la zone II. Il est flagrant que la déformation de la courbe à ce niveau est différente après injections de FSH chez un animal immature hypophysectomisé et chez un animal immature sensibilisé par HCG. Dans le premier cas, FSH modifie la courbe de fréquence des tailles de follicules par déplacement du mode du pic de la zone II vers les plus grandes tailles. Dans le second, la courbe se déforme par écrasement du pic de la zone II sans déplacement du mode de la courbe. Pour comprendre ces différentes déformations des courbes, il faut faire appel aux courbes de fréquence établies avec la même méthodologie d'analyse chez les animaux immatures de même âge, hypophysectomisés et intacts et suivre alors les déformations des courbes pendant le même laps de temps de 4 jours.

Chez l'animal immature hypophysectomisé, il existe encore des follicules de la zone III. L'atrésie va les faire disparaître et frapper surtout dans les 4 jours suivants les follicules de la partie droite du pic de la zone II (BENOIT, I967). Or, après injection de FSH, le nombre total de follicules de toutes tailles augmente de $50 \mathrm{p}$. Ioo par rapport à celui de l'animal non traité. Le pourcentage de follicules atrétiques diminue de 39 p. Ioo à I 8 p. Ioo dans les mêmes conditions. FSH empêche donc la moitié des follicules d'involuer. La modification de la forme de la courbe au niveau du pic de la zone II est donc d'abord le reflet de cette diminution de l'involution; mais il peut être aussi celui d'une augmentation des espaces intracellulaires qui sont réduits dans les follicules de la ratte immature hypophysectomisée (BENOIT, I967). I1 faut cependant admettre qu'il y a transfert de follicules de cette zone II vers la zone III puisque pour des doses élevées de FSH injectées, le nombre de follicules de la zone III est trop grand pour ne provenir que de la suppression de l'involution de 
follicules. L'absence d'écrasement du pic prouve que le transfert de follicules de la zone II vers la zone III doit être compensé par transfert de follicules de la zone I vers la zone II. Cette dernière hypothèse est compatible avec 1'action de FSH sur le taux de multiplication des cellules folliculeuses signalé dans le paragraphe précédent pour les follicules de cette taille (zone I).

Chez l'animal immature intact traité selon STEELMAN et POHLEy, le nombre total de follicules n'est pas modifié après traitement de 4 jours par FSH. La diminution de l'involution des follicules est donc un effet mineur de FSH. En revanche, il y a un important transfert de follicules de la zone I vers la zone II d'où écrasement du pic de la courbe dans la zone II. Aucune compensation n'existe venant d'un transfert de follicules de la zone I. Une telle modification n'existe pas entre les courbes de fréquences des populations folliculaires d'ovaires de ratte témoin de 24 jours et de 28 jours. Mais ce même type de déformation de courbe a été constaté chez la femelle immature entre le $20^{\mathrm{e}}$ et le $24^{\mathrm{e}}$ jour lorsque se produit une importante décharge de FSH à partir de l'hypophyse (MARIANA et al., I972).

Ces différences de compensation dans les passages des follicules d'une zone vers une autre après injection de FSH selon qu'il s'agit de ratte hypophysectomisée ou intacte reflètent des différences dans l'involution préexistante au traitement FSH. Mais elles soulignent aussi des modifications de vitesses de croissance plus marquée chez l'animal hypophysectomisé que chez l'animal intact. PEDERSEN (I959) a signalé que la vitesse de croissance variait pour une catégorie folliculaire donnée avec l'âge de l'animal. Il est vraisemblable que des variations de l'équilibre hormonal en sont la cause.

\title{
REMERCIEMENTS
}

Les auteurs remercient M. O. Moulin pour son assistance technique.

Ils remercient le National Institute of Health pour leur don de FSH NIH S et de GH NIH S ainsi que M. M. Jutisz pour le don de $\mathrm{LH} \mathrm{M}_{1}$ purifiée dans son laboratoire.

\section{SUMMARY}

\author{
EFFECT OF HYPOPHYSEAL GONADOTROPHINS \\ ON OVARIAN FOLLICULAR POPULATION IN THE IMMATURE RAT
}

The quantitative changes in the ovarian follicular population are analyzed on the frequency curve of follicles classified by size criteria. The curve is divided into three zones (I + II + III) related to three steps of follicular growih. Two types of immature rats are used : hypophysectomized rats after seven days of ovarian regression (EvANs and SIMPSON, rat) and intact and HCG sensitized rats (Steelman and Pohley, rat). The FSH assays are done with these two types of rats.

In these two physiological conditions, the third zone curve (follicles with antra) first rises. The area of the stimulated follicles (size of follicles $\times$ number of follicles of each size) increases proportionally with the dose of FSH. This the FSH assays really measure the development of follicles with antra. In these conditions this particular effect is specific for FSH. GH, LH and prolactin do not induce the appearance of antrum follicles in immature hypophyzectomized rat.

FSH also changes the other two zones ( $I$ and II) of the frequency curve : the relative and absolute numbers of these growing follicles increase in the EvaNs rat and decrease in the STEELMANPolmey rat. Such an effect is not specific, GH and prolactin also act on the follicles of these zones. 
The interaction of FSH and LH is particularly evident throughout follicular growth and exerts a homothetic effect on the frequency curve.

The action of FSH on evolution and involution rates is not the same in the two endocrine situations. It is possible that the hypophyseal hormones affect different stages of follicular growth. These two points are discussed.

\section{RÉFÉRENCES BIBLIOGRAPHIQUES}

AвеrсRombie M., 1964. Estimation of nuclear population from microtome sections. Anat. Rec., 94, 239-247.

Baker T. G., Neal P., I972. Initiation and control of meiosis and follicular growth in ovaries of the mouse. Coll. Ovogenèse-Folliculogenèse, Nouzilly, France, 14-I5/12/72.

BENorT M., I967. Etude des populations folliculaires d'ovaires de rattes impubères et hypophysectomisées. Diplôme d'études supérieures, Univ., Paris.

Evans H. M., Simpson M. E., Tolksborf S., Jensen H., r939. Biological studies of the gonadtropic principles in sheep pituitary substance. Endocrinology, 25, 529-546.

FalcoNi G., Rossi G. L., I964. Transauricular hypophysectomy in rats and mice. Endocrinology, 74, 301-303.

Hrsaw F. L., I947. Development of the graafian follicle and ovulation. Physiol. Rev., 27, 95-Ir9.

Lostroh A. J., Johnson R. E., 1966. Amounts of interstitial cell stimulating hormone and follicle stimulating hormone required for follicular development, uterine growth and ovulation in the hypophysectomized rat. Endocrinology, 79, $991-996$.

Mariana J.-C., I972. Classification des follicules ovariens : principes et méthodes d'étude. Ann. Biol. anim. Bioch. Biophys., 12, 377-382.

Mariana J.-C., DE Reviers M.-M., Mauléon P., I972. Some endocrine parameters in three lines of rats. Cong. The Development and Maturation of the Reproductive organs and Functions in the female. Copenhague, $I-2$ july 72 .

Mauléon P., Hanumantha Rao K., I963. Variations génétiques des populations folliculaires dans les ovaires de rattes impubères. Ann. Biol. anim. Bioch. Biophys., 3, 2I-3I.

Maulton P., Benort M., r964. Influences génétiques et endocriniennes sur les populations folliculaires ovariennes mises en évidence par une nouvelle méthode d'étude. Ve Cong. intern. Reprod. anim. Insém. artif., Trente, 425-43I.

Pedersen T., 1969. Follicles growth in the immature mouse ovary. Acta Endocr., 62, II7-I32.

PEDERSEN T., I970. Determination of follicle growth rate in the ovary of the immature mouse. $J$. Reprod. Fert., 21, 8 I-93.

RYLE M., I972. The growth in vitro of mouse ovarian follicles of different sizes in response to purified gonadotrophins. J. Reprod. Fert., 30, 395-405.

Steflman S. L., Pohley F. M., 1953. Assay of the follicle stimulating hormone based on the augmentation with human chorionic gonadotrophin. Endocrinology, 53, 604-6I6. 